\title{
Management of Malignants Gliomas: About 20 Cases Treated in the Medical Oncology Department of Fattouma Bourguiba University Hospital-Monastir
}

\author{
Hiba Sboui", Amira Daldoul, Nader Slema, Amal Chamsi, Sonia Zaied \\ Medical Oncology Department, Fattouma Bourguiba Hospital, Monastir, Tunisia \\ Email: *sboui-hiba2006@hotmail.com, amira.krir@outlook.com, slama.nader85@gmail.com, chamsiamal90@gmail.com, \\ soniazaied@gmail.com
}

How to cite this paper: Sboui, H., Daldoul, A., Slema, N., Chamsi, A. and Zaied, S. (2021) Management of Malignants Gliomas: About 20 Cases Treated in the Medical Oncology Department of Fattouma Bourguiba University Hospital-Monastir. Journal of Cancer Therapy, 12, 478-485.

https://doi.org/10.4236/jct.2021.128041

Received: July 19, 2021

Accepted: August 22, 2021

Published: August 25, 2021

Copyright $\odot 2021$ by author(s) and Scientific Research Publishing Inc. This work is licensed under the Creative Commons Attribution International License (CC BY 4.0).

http://creativecommons.org/licenses/by/4.0/

\section{(c) (i) Open Access}

\begin{abstract}
Introduction: Malignant gliomas refer to grade III or IV brain tumors defined according to the World Health Organization (WHO) classification. They are a heterogeneous group of pathologies and represent a serious health problem by their frequency, severity and treatment difficulties. The prognosis of malignant gliomas remains poor despite all the medical advances. Materials and Methods: It is a retrospective study included 20 cases of malignant glial tumors treated at the medical oncology department, Fattouma Bourguiba hospital in Monastir between 2012 and 2016, according to the STUPP protocol. Results: These were 12 men and 8 women with a median age of 43 . Clinical signs were not very specific, dominated by intracranial hypertension and deficit signs. Imagery referred to the diagnosis of malignant gliomas in 1st intention. Surgery consisted of a macroscopically complete exeresis in (15\%) cases, a partial exeresis in (50\%), the rest of the patients had a stereotactic biopsy. Histology found GBM in 16 patients (80\%), 2 cases of Grade III anaplastic astrocytoma (10\%), 1 case of anaplastic oligodendroglioma (5\%), and 1 case of Grade III anaplastic eppendymoma (5\%). Most of our patients received concurrent radio-chemotherapy and adjuvant TMZ chemotherapy was administered in 15 patients, 7 of whom received the full 6 scheduled cures. A relapse treatment was decided in only one of the 12 patients who relapsed. 6 patients are still alive. The median survival is 11.27 months. In our series, overall survival was related to histological type $(p=0.006)$ and neurological status assessed at the end of RT-CT $(p=0.001)$. While age, general condition score, type of surgery, and post-therapeutic development did not show a sta-
\end{abstract}


tistically significant relationship, although survival rates were consistent with the criteria assessed. Conclusion: Malignant gliomas are rare tumors, bad prognosis, aggravated in Tunisia by a diagnostic delay. The creation of a multidisciplinary neuro-oncology group can help to improve management.

\section{Keywords}

Malignant Gliomas, Surgery, Radiotherapy, Chemotherapy, Prognosis

\section{Introduction}

High-grade gliomas (GHGs) refer to brain tumors of Grade III or IV defined histologically according to the latest WHO World Health Organization classification of 2016 [1].

This is a heterogeneous group including glioblastoma and its variants (WHO grade IV), anaplastic astrocytoma and its subtypes (WHO grade III), and anaplastic oligodendroglioma (WHO grade III) [1].

Malignant gliomas remain the major problem of neuro-oncology, due to their frequency, severity and the difficulties of treatment they pose.

Despite all the diagnostic and therapeutic advances, the prognosis of malignant gliomas is unfortunately still bleak in the short and medium term. Despite a multidisciplinary therapeutic management based on surgery, radiotherapy and alkylating agents, the median survival of patients with malignant glioma is in the order of 12 to 14 months [2].

Faced with the particular aspect of this type of tumor, we carried out this work to determine the epidemiological, diagnostic aspects of malignant glial tumors and their therapeutic management. The aim of this work is also to formulate recommendations adapted to the surgical and medical therapeutic possibilities available in Tunisia.

\section{Methods}

This is a retrospective study spread over a period of 4 years from March 2012 to December 2016. This study includes 20 patients treated at the medical department of Fattouma Bourguiba Hospital in Monastir (Tunisia), having a histological diagnosis of malignant glioma, and having received medical treatment according to the STUPP protocol.

The data collection was based on the data from the patient files monitored at the Medical Carcinology Unit and the Neurosurgery Department CHU Fattouma Bourguiba de Monastir, on an information sheet containing the following information: patient identification, initial clinical symptomatology, morphological data, histological data, therapeutic management, patient evolution.

The statistical analysis was performed using the SPSS 20.0 software. Overall survival was calculated from the date of histological diagnosis to death or the latest news using the Kaplan-Meier method. The comparison of different surviv- 
al rates was based on the log-RANK test. A value of $\mathrm{p}<0.05$ is considered statistically significant.

The literature review focused on indexed articles in the bibliographic computer databases "Medline", "Cochrane database”, "Science direct”, "Google scholar", using the keywords derived from the Mesh terminology: "High-grade Gliomas, Glioblastoma, Astrocytoma, Oligodendrioglioma, epidemiology, survival, treatment, radiotherapy, chemotherapy" published in English or French.

We selected the most recent articles and those of the major retrospective series or with a satisfactory level of proof (Phase II - III or meta-analyzes) important for the discussion of this work.

The management of bibliographic references was done using the Zotero software.

\section{Results}

Our study included 20 cases of malignant glial tumors. The median age was 43 years with male predominance $(S R=1.5)$. The average time for diagnosis was 64 days. All the patients were neurologically symptomatic.

Signs of intracranial hypertension, neurological deficit and epilepsy dominated the clinical picture with some case of behavioral disorder.

Eighty percent $(80 \%)$ of our patients had good overall condition (WHO $=1$ ).

Imaging referred to the 1st-line diagnosis and revealed a unifocal lesion in $65 \%$ of patients, bi or multifocal in $35 \%$ of cases. The seat was temporal in $24.1 \%$ (7 patients), frontal in 31\% (2 patients), parietal in 31\% (6patients) and occipital in $10.3 \%$ (2 Patient). The location was more right (14 cases) than left ( 6 cases). Tumor lesion was hypo-signaled on T1-weighted sequences in 12 cases (60\%), hyper-signaled in 3 patients (15\%), iso-signaled in 1 case (5\%), and heterogeneous signaled in one patient (5\%). On T2-weighted sequences, the majority of lesions were hyper-signaled in 17 patients (85\%) (Figure 1).

Ten patients had a partial resection (50\%), a macroscopically complete exeresis for 3 patients (15\%) and stereotaxic biopsy was performed in 7 patients $(35 \%)$.

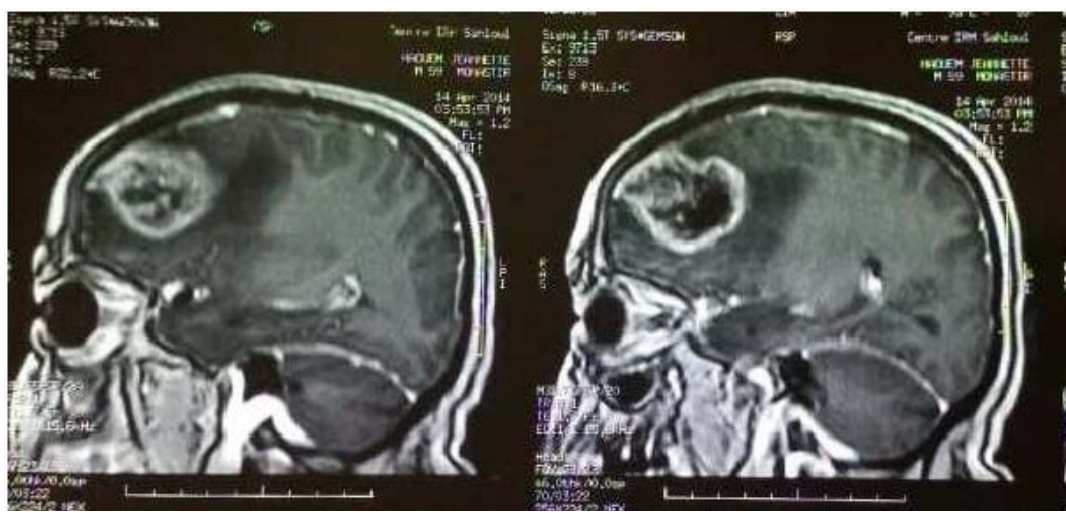

Figure 1. A case of multiform Glioblastoma. 
Histology confirmed the diagnosis of high-grade glioma; 16 patients had a multiform glioblastoma ( $80 \%$ of cases), a Grade III anaplastic astrocytoma was objectified in 2 patients (10\%), a Grade III oligodendroglioma case (5\%), and a Grade III anaplastic ependomoma case (5\%). No patient has had a molecular study.

Radiation therapy was initiated within an average of 52 days after surgery and was performed for the majority of patients by modern Cobalt (ELITE 100).

Seventeen patients had received TMZ, 12 of which had started on the first day of radiotherapy, while the remaining patients had received TMZ (5 cases), chemotherapy was initiated during irradiation.

Only 15 patients received adjuvant TMZ treatment. It started after the end of the RT-CT with an average delay of 55 days, ranging from 30 to 90 days. Eight patients had received the 6 cures prescribed by the STUPP protocol. Three patients had received 4 cures and the remaining 4 patients had only 3 cures (deaths before completing the protocol).

The median survival from histological diagnosis to the date of death or the latest news was 11.27 months ranging from 1.17 to 43.5 months. It decreased with age $(p=0.004)$. She was significantly better in case of complete surgical excision ( 9 to 34 months; $\mathrm{p}<0.0001$ ), postoperative radiotherapy (13 to 16 months; $\mathrm{p}=0.025$ ), and postoperative chemoradiotherapy (11 to 34 months; $\mathrm{p}<$ $0.0001)$.

Overall survival at 1 year was $45 \%$ (Figure 2).

Twelve patients in our study had a tumor progression, or $80 \%$ of cases, of which only one patient was treated with TMZ after 10 months of adjuvant CT for 3 cycles. While therapeutic abstention and the use of palliative care was the decision of neuro-carcinologists for the rest of the patients (11 patients). Six patients in our series were still alive and two were lost in the study.

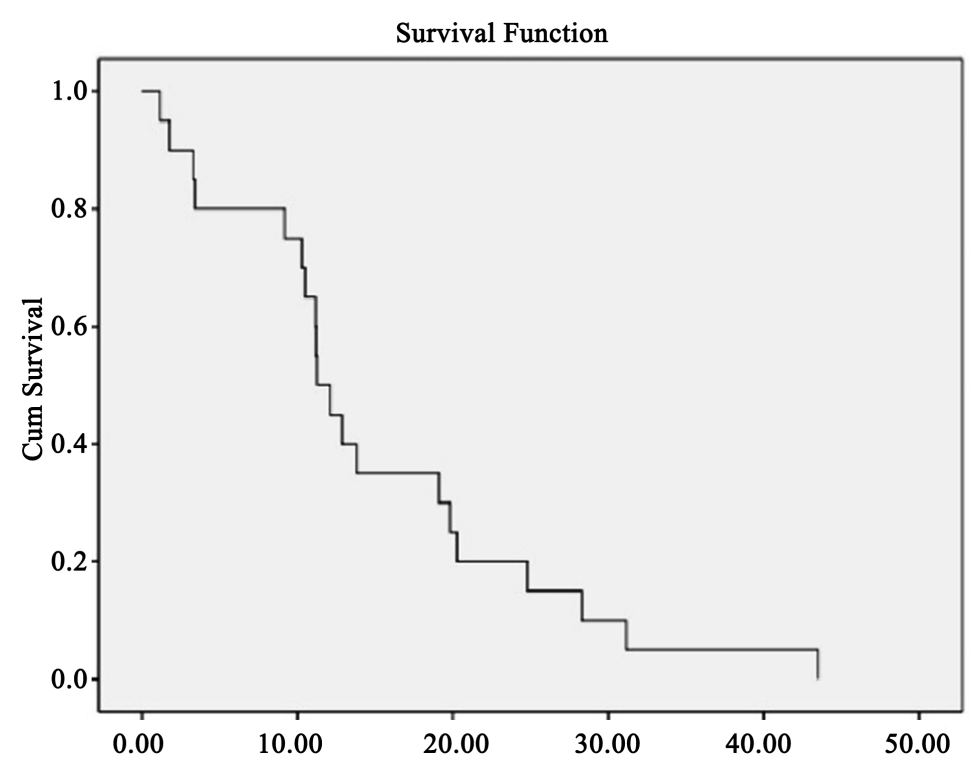

Figure 2. Survival function. 


\section{Discussion}

Our study examined a series of 20 cases of histologically confirmed malignant glial tumors. Multiform glioblastoma is the most common histological type (80\% of cases).

The diagnosis was relatively long by 64 days. This results in clinical scalability for our patients, such as the neurological deficit in $35 \%$ of cases and the impact on the general condition.

Imaging based on MRI and CT enabled in all cases the diagnosis of glial tumor and the prediction of its degree with a good reliability.

Surgical care consisted in the majority of cases of partial resection (50\%). Radiation therapy was initiated within an average of 52 days of surgery.

Seventeen patients had received Témozolomide (TMZ) under the STUPP protocol.

Fifteen patients received adjuvant TMZ treatment. Twelve patients had tumor progression, or $80 \%$ of the cases. Overall survival at 1 year was $45 \%$.

Malignant gliomas remain the major problem of neuro-oncology.

Despite all the diagnostic and therapeutic advances, most patients die within three years of diagnosis. Given the seriousness of this type of tumors, and the disappointing therapeutic results, we have carried out this work in order to determine the different aspects of malignant gliomas and to deduce recommendations adapted to the surgical and medical therapeutic possibilities available in Tunisia.

The epidemiological profile of our series was consistent with international data [3] [4].

Histopathological evidence is required to characterize the tumor grade, and it must be supplemented by a molecular study to define the glioma subtype according to the revised classification of tumors of the central nervous system of the World Health Organization published in 2016 [1]. These molecular studies have a prognostic and predictive of therapeutic response.

Intracranial hypertension is the most frequent sign, associated with localization signs according to the anatomical site reached [5]. The severity and severity of the initial clinical signs, by their impact on the general condition, can condition the prognosis, and that the remaining negative factors reported by the majority of authors [6].

MRI plays a key role in the management of malignant gliomas. This radiological reference technique with its diffusion, infusion and spectroscopy sequences, has a considerable contribution to make the diagnosis, to guide as precisely as possible the surgical gesture but also to delimit the functional areas close to the tumor and the target volume of the radiotherapy, without forgetting the posttherapeutic follow-up [7] [8] [9] [10].

Surgical treatment is based on the fullest possible tumor resection while preserving the cognitive functions and quality of life of patients.

The extent of resection is correlated with overall survival. Indeed, patients with a complete exeresis without residual fluorescence have a median of SG at 20.9 months compared to 12.3 months $(\mathrm{p}=0.000559)$ in the case of incomplete 
exeresis [11].

Based on the surgical data in our series and comparing it to the literature, the surgical objective is only achieved in $20 \%$ to $49 \%$ of cases, in particular because of the infiltrating character of malignant gliomas [11].

RT with TMZ (STUPP protocol) is the treatment of choice for glioblastoma [12] [13]. With regard to anaplastic oligodendroglioma, TMZ is the preferred diet over the PCV protocol, due to its ease of administration and its better tolerance [14]. However, PCV remains an option for anaplastic oligodendroglial tumors [15] [16].

Our results are not as good as literature, the number of patients is limited, but we believe that our support is generally satisfactory considering the means available in Tunisia and we recommend:

- The creation of a multidisciplinary group of neuro-oncology associating; neurosurgeons, radiologists, anatomopathologists, medical oncologists and radiotherapists.

- The establishment of a molecular biology platform to follow the new recommendations and of course to enable patients to benefit from treatment "Custom".

- Care to preserve the quality of life of patients and their families must be included in the therapeutic decision.

- Use of support and end-of-life support should be an integral part of care.

\section{Conclusion}

The prognosis of malignant gliomas remains bleak despite all medical progress with survival medians of 12 to 15 months for the GBM and about 3 years for the other gliomas of grade III. Management must be multidisciplinary, involving neurosurgeons, radiologists, anatomopathologists, medical oncologists and radiotherapists, as soon as the diagnosis is suspected. In Tunisia, several measures must be put in place to improve the management of malignant gliomas.

\section{What Is Known about This Subject}

- Malignant gliomas remain the major problem of neuro-oncology in the world, due to their frequency, severity and the difficulties of treatment they pose.

- Despite a multidisciplinary treatment, the prognosis of malignant gliomas remains bleak.

- Few data on malignant gliomas are available in Tunisia.

\section{What Does Your Study Bring back}

- Determine the epidemiological, diagnostic aspects of malignant glial tumors and their therapeutic management in tunisia.

- Develop recommendations adapted to the surgical and medical therapeutic possibilities available in Tunisia.

\section{Conflicts of Interest}

The authors do not declare any conflict of interest. 


\section{References}

[1] Louis, D.N., Perry, A., Reifenberger, G., von Deimling, A., Figarella-Branger, D., Cavenee, W.K., et al. (2016) The 2016 World Health Organization Classification of Tumors of the Central Nervous System: A summary. Acta Neuropathologica, 131, 803-820. https://doi.org/10.1007/s00401-016-1545-1

[2] Phuong, C.P., Nam, V.L., Schild, E.S., Rades, D. and Khoa, T.M. (2017) A Survival Score Based on Symptoms and Performance Status for Patients with High-Grade Gliomas Receiving Radiochemotherapy. In Vivo, 31, 689-693.

https://doi.org/10.21873/invivo.11114

[3] Ostrom, Q.T., Gittleman, H., Fulop, J., Liu, M., Blanda, R., Kromer, C., et al. (2015) CBTRUS Statistical Report: Primary Brain and Central Nervous System Tumors Diagnosed in the United States in 2008-2012. Neuro-Oncology, 17, iv1-iv62. https://doi.org/10.1093/neuonc/nov189

[4] Ostrom, Q.T., Bauchet, L., Davis, F.G., Deltour, I., Fisher, J.L., Langer, C.E., et al. (2014) The Epidemiology of Glioma in Adults: A "State of the Science" Review. Neuro-Oncology, 16, 896-913. https://doi.org/10.1093/neuonc/nou087

[5] Altwairgi, A.K., Raja, S., Manzoor, M., Aldandan, S., Alsaeed, E., Balbaid, A., et al. (2017) Management and Treatment Recommendations for World Health Organization Grade III and IV Gliomas. International Journal of Health Sciences, 11, 54-62.

[6] Posti, J.P., Bori, M., Kauko, T., Sankinen, M., Nordberg, J., Rahi, M., et al. (2015) Presenting Symptoms of Glioma in Adults. Acta Neurologica Scandinavica, 131, 88-93. https://doi.org/10.1111/ane.12285

[7] Martin-Duverneuil, N., Guillevin, R. and Chiras, J. (2008) Imagerie des gliomes. Cancer Radiothérapie, 12, 669-675. https://doi.org/10.1016/j.canrad.2008.07.017

[8] Bracard, S., Taillandier, L., Antoine, V., Kremer, S., Taillandier, C. and Schmitt, E. (2006) Cerebral Gliomas: Imaging Diagnosis and Follow-Up. Japanese Journal of Radiology, 87, 779-791. https://doi.org/10.1016/S0221-0363(06)74087-2

[9] Boxerman, J.L., Shiroishi, M.S., Ellingson, B.M. and Pope, W.B. (2016) Dynamic Susceptibility Contrast MR Imaging in Glioma: Review of Current Clinical Practice. Magnetic Resonance Imaging Clinics of North America, 24, 649-670. https://doi.org/10.1016/j.mric.2016.06.005

[10] Dhermain, F.G., Hau, P., Lanfermann, H., Jacobs, A.H. and van den Bent, M.J. (2010) Advanced MRI and PET Imaging for Assessment of Treatment Response in Patients with Gliomas. Lancet Neurology, 9, 906-920. https://doi.org/10.1016/S1474-4422(10)70181-2

[11] Jacquesson, T., Ducray, F., Maucort-Boulch, D., Armoiry, X., Louis-Tisserand, G., Mbaye, M., et al. (2013) Surgery of High-Grade Gliomas Guided by Fluorescence: A Retrospective Study of 22 Patients. Neurochirurgie, 59, 9-16. https://doi.org/10.1016/j.neuchi.2012.07.002

[12] Feuvret, L., Antoni, D., Biau, J., Truc, G., Noël, G. and Mazeron, J.-J. (2016) Référentiels d'irradiation des gliomes. Cancer Radiothérapie, 20, S69-S79.

https://doi.org/10.1016/j.canrad.2016.07.008

[13] Stupp, R., Mason, W.P., van den Bent, M.J., Weller, M., Fisher, B., Taphoorn, M.J., et al. (2005) Radiotherapy plus Concomitant and Adjuvant Temozolomide for Glioblastoma . The New England Journal of Medicine, 352, 987-996. https://doi.org/10.1056/NEJMoa043330

[14] Ducray, F., Sierra del Rio, M., Carpentier, C., Psimaras, D., Idbaih, A., Dehais, C., et al. (2011) Up-Front Temozolomide in Elderly Patients with Anaplastic Oligodendroglioma and Oligoastrocytoma. Journal of Neuro-Oncology, 101, 457-462. 
https://doi.org/10.1007/s11060-010-0264-Z

[15] Van den Bent, M.J., Carpentier, A.F., Brandes, A.A., Sanson. M,, Taphoorn, M.J., Bernsen, H.J., et al. (2006) Adjuvant Procarbazine, Lomustine, and Vincristine Improves Progression-Free Survival but Not Overall Survival in Newly Diagnosed Anaplastic Oligodendrogliomas and Oligoastrocytomas: A Randomized European Organisation for Research and Treatment of Cancer Phase III trial. Journal of Clinical Oncology, 24, 2715-2722. https://doi.org/10.1200/JCO.2005.04.6078

[16] Van den Bent, M.J., Brandes, A.A., Taphoorn, M.J.B., Kros, J.M., Kouwenhoven, M.C.M., Delattre, J.-Y., et al. (2013) Adjuvant Procarbazine, Lomustine, and Vincristine Chemotherapy in Newly Diagnosed Anaplastic Oligodendroglioma: LongTerm Follow-Up of EORTC Brain Tumor Group Study 26951. Journal of Clinical Oncology, 31, 344-350. https://doi.org/10.1200/JCO.2012.43.2229 